\title{
The blending of NBR/EPDM with montmorillonite as compatibilizer: The effect of different accelerator
}

\author{
Hesty Eka Mayasari ${ }^{1 *}$, Agung Yanuar Wirapraja ${ }^{1}$, Ike Setyorini ${ }^{2}$ \\ ${ }^{1}$ Center of Industrial Research and Standardization of Surabaya, Jl. Jagir Wonokromo 360, Surabaya 60244, Indonesia \\ ${ }^{2}$ Center for Leather, Rubber and Plastics, Jl. Sokonandi 9, Yogyakarta 55166, Indonesia \\ * Corresponding author. Tel.: +62 3199843670 , Fax.: +62 318410480 \\ E-mail: hestyeka@kemenperin.go.id
}

Submitted: 11 February 2020

Revised: 2 May 2020

Accepted: 4 May 2020

\begin{abstract}
The rubber blend is widely used in industry because of its excellent properties. In this study, Nitrile Butadiene Rubber (NBR) was blended with Ethylene Propylene Diene Monomer (EPDM) rubber using montmorillonite as the compatibilizer. Various kinds of accelerator from different accelerator group (DPG, MBT, CBS, TMTD, and ZDEC) were employed into this blend. This research aimed to study the effect of different accelerators on the curing characteristic, mechanical, and swelling properties of the NBR/EPDM blends. The result findings showed that DPG gave the longest scorch and optimum curing time. DPG also gave the lowest torque difference, cure rate index, and also rate constant. Meanwhile, TMTD gave the fastest optimum curing time and also the highest torque difference, cure rate index, and rate constant. The other accelerators gave the curing characteristic among them. The MBT gave the highest tensile, elongation, and tear strength. Based on this research, the TMTD, as secondary accelerator, was the suitable accelerator for improving the curing characteristic property and MBT, as primary accelerator, for improving the mechanical properties of NBR/EPDM blend.
\end{abstract}

Keywords: accelerator, compatibilizer, montmorillonite, NBR/EPDM.

\section{INTRODUCTION}

Nowadays, researchers are interested in polymeric materials obtained by blending two or more rubbers. The blending of rubber can improve the material's properties. The weakness of the single rubber can be improved by the superiority of another rubber. The rubber blend is preferred in the industry because it is easier to be processed and has good mechanical properties (Jovanović et al., 2013). Nitrile butadiene rubber (NBR) has a high resistance to solvent and oil, but it has poor resistance to ozone and heat aging. Ethylene propylene diene monomer (EPDM) rubber has a high resistance to ozone and heat aging, but it has poor resistance to solvent. The blending of NBR and EPDM can produce the composite with the best properties from each component. The composite can have excellent resistance to oil and solvent and also have excellent resistance to ozone and heat aging (Jovanović et al., 2013; Mayasari \& Setyadewi, 2018). This composite can be used as a seal, gasket, hoses, transmission belts, and also conveyor belts.

One of the important aspects in rubber blending is the compatibility of the rubbers. Different polarity and solubility can make an incompatible blend and affect the rubber properties. Compatibilizer is needed to achieve the compatible blend such as maleic anhydride (Bonilla-Cruz et al., 2017), silica (Kapgate \& Das, 2014), montmorillonite, bentonite (Muniyadi \& Ismail, 2013), and methacrylate butadiene styrene (Mayasari \& Setyadewi, 2018). Montmorillonite is a kind of silica that has unique properties. Montmorillonite has a layer composed by a silica tetrahedral layer sandwiched between the two octahedral sheets of magnesium hydroxides and aluminium (Azizli et al., 2018). The previous studies have proved that montmorillonite could act as good compatibilizer for rubber composite. Yi et al. (2014) found that montmorillonite could improve the mechanical and flame-retardant 
Table 1. Formulation of NBR/EPDM composite.

\begin{tabular}{lrrrrr}
\hline \multirow{2}{*}{ Materials } & \multicolumn{5}{c}{ Composite } \\
\cline { 2 - 6 } & NEM1 & NEM2 & NEM3 & NEM4 & NEM5 \\
\cline { 2 - 6 } & 75.0 & 75.0 & 75.0 & 75.0 & 75.0 \\
\hline NBR & 25.0 & 25.0 & 25.0 & 25.0 & 25.0 \\
EPDM & 5.0 & 5.0 & 5.0 & 5.0 & 5.0 \\
Zinc Oxide & 1.0 & 1.0 & 1.0 & 1.0 & 1.0 \\
Stearic acid & 30.0 & 30.0 & 30.0 & 30.0 & 30.0 \\
HAF CB N330 & 30.0 & 30.0 & 30.0 & 30.0 & 30.0 \\
SRF CB N774 & 5.0 & 5.0 & 5.0 & 5.0 & 5.0 \\
Montmorillonite & 10.0 & 10.0 & 10.0 & 10.0 & 10.0 \\
Paraffinic oil & 0.5 & 0.5 & 0.5 & 0.5 & 0.5 \\
Paraffinic wax & 5.0 & 5.0 & 5.0 & 5.0 & 5.0 \\
6 PPD Vulkanox & 2.0 & - & - & - & - \\
DPG & - & 2.0 & - & - & - \\
MBT & - & - & 2.0 & - & - \\
CBS & - & - & - & 2.0 & - \\
TMTD & - & - & - & - & 2.0 \\
ZDEC & 1.5 & 1.5 & 1.5 & 1.5 & 1.5 \\
Sulfur & & & & \\
\hline
\end{tabular}

*phr: part per hundred rubber

properties of EPDM composite. Sun et al. (2013) found that organic montmorillonite could improve the curing characteristic properties of chloroprene rubber (CR) composite. Nik Ismail et al. (2014) found that montmorillonite could improve the thermal properties of silicone rubber.

The accelerator has an important function in the rubber blends. It can increase the speed of the curing process. Different accelerator gave different effects on rubber composite (Nabil et al., 2014). There were some previous research studied about the effect of accelerator on the rubber composite, such as in EPDM/NR composite (Mayasari et al., 2019), NR/CR composite (Azar \& Sen, 2017), and NBR composite (Movahed et al., 2015). However, based on the author's knowledge, there has been no research on the effect of the different accelerators on the NBR/EPDM composite using montmorillonite as compatibilizer.

The aim of this study was to investigate the effect of the five single different accelerators from different accelerator group on NBR/EPDM composite using montmorillonite as compatibilizer. The accelerators applied in this study included primary accelerators (2-mercaptobenzothiazole and n-cyclohexyl-2-benzothiazole sulfenamide) and secondary accelerators (diphenyl guanidine, tetramethyl thiuram disulphide), zinc diethyldithiocarbamate). The response of each accelerator on curing characteristics, mechanical and swelling properties of the NBR/EPDM blends will be evaluated and discussed.

\section{MATERIALS AND METHODS Materials}

The rubbers used in this study were NBR (ACN 48.5\%, Krynac 4975 F) and EPDM (ENB 8.7\%, Keltan 4551 A). Carbon black (CB) N330 High Abrasion Furnace (HAF) and N774 Semi Reinforcing Furnace (SRF) (OCI, Korea) were used as filler, while montmorillonite (Sigma Aldrich) as the compatibilizer. The paraffinic oil (Indrasari) was used as a softener, paraffin wax (Antilux 654 A) and N-(1,3-dimethylbutyl)-N'phenyl-p-phenylendiamine (6PPD, Starchem) were used as the antioxidant. Aflux $42 \mathrm{M}$ (Rhein Chemie) and zinc oxide ( $\mathrm{ZnO}$, Indoxide) were used as the activator. The sulfur (Miwon) was used as vulcanizing agent. The diphenyl guanidine (DPG, Shandong Sianxian), 2-mercaptobenzothiazole (MBT, Bayer), n-cyclohexyl-2-benzothiazole sulfenamide (CBS, Kemai), tetramethylthiuram disulfide (TMTD, Shandong Sianxian), and zinc diethyldithiocarbamate (ZDEC, Shandong 
Sianxian) were used as the accelerator.

\section{Methods}

\section{NBR/EPDM blends preparation}

The rubbers and the additives were mixed using a two-roll mill according to the formulation listed in Table 1. Then, the resulted NBR/EPDM compounds were allowed to rest for at least 24 $\mathrm{h}$ in a dry conditioned room before subsequent processes.

\section{Curing characteristic}

The curing characteristics of the blends were tested at $160^{\circ} \mathrm{C}$ using the Moving Die Rheometer (MDR) Gotech M-3000A. The extracted data included the minimum $\left(M_{L}\right)$ and maximum $\left(M_{H}\right)$ torques, scorch time $\left(\mathrm{ts}_{2}\right)$, and optimum curing time $\left(t_{90}\right)$. These data were useful for preparing the vulcanized test pieces and assessing the curing behavior of the resulted blends. The cure rate index (CRI), in $\mathrm{min}^{-1}$, could be calculated using equation (1)

$C R I=\frac{1}{\left(t_{90}-t s_{2}\right)}$

\section{Curing kinetic}

The curing kinetic of the NBR/EPDM compound represents the kinetic reaction during the curing process. The rate constant $(k)$ can be calculated from rheometer data using equation (2) (Mayasari et al., 2019). The rate constant $(k)$ was obtained from the plot of $\ln \left(M_{H}-M_{t}\right)$ versus $t$.

$$
\ln \frac{\left(M_{H}-M_{L}\right)}{\left(M_{H}-M_{t}\right)}=k t
$$

Hence, by rearranging the Equation (2), obtained Equation (3)

$\ln \left(M_{H}-M_{t}\right)=\ln \left(M_{H}-M_{L}\right)-k t$
Where $M_{H}$ as the maximum torque (kgf.cm), $M_{L}$ as the minimum torque (kgf.cm), $M_{t}$ as the torque at a certain time (kgf.cm), $t$ as the time (s), and $k$ as the rate constant $\left(\mathrm{min}^{-1}\right)$. The rate constant $(k)$ could be obtained from the slope of the line through plotting $\ln \left(M_{H}-M_{t}\right)$ against the time $(t)$. The reaction was assumed as a first order reaction in this study. This study also estimated $M$ values at $25 \%$ until $45 \%$ of torque change, because it was assumed that the curing process is mostly happening at that time, hence it could represent the rate constant rate (Nampitch \& Buakaew, 2006).

\section{Mechanical properties}

The tensile strength, elongation of break, and the tear strength of the NBR/EPDM composite were studied using the tensile strength tester Kao Tieh Tensile strength, and elongation were measured in accordance to ISO 37, while tear strength was following ISO 34.

\section{Swelling property}

The swelling tests were carried out according to ISO 1817. Specimens with thickness $(2 \pm 0.2)$ $\mathrm{mm}$ were immersed in IRM 903 at ambient temperature for $72 \mathrm{~h}$. The change in mass in noted and the swelling percentage was calculated using Equation (4). Where $w_{l}$ is the mass after swelling and $w_{0}(\mathrm{~g})$ is the initial mass $(\mathrm{g})$.

Swelling $(\%)=\left(\frac{w_{1}-w_{0}}{w_{0}}\right) \times 100$

\section{RESULTS AND DISCUSSION Cure Characteristic}

Figure 1 shows the torque changing of the NBR/EPDM blends on various accelerators during vulcanization. Generally, the curing curve is divided into three regions, i.e. induction, curing

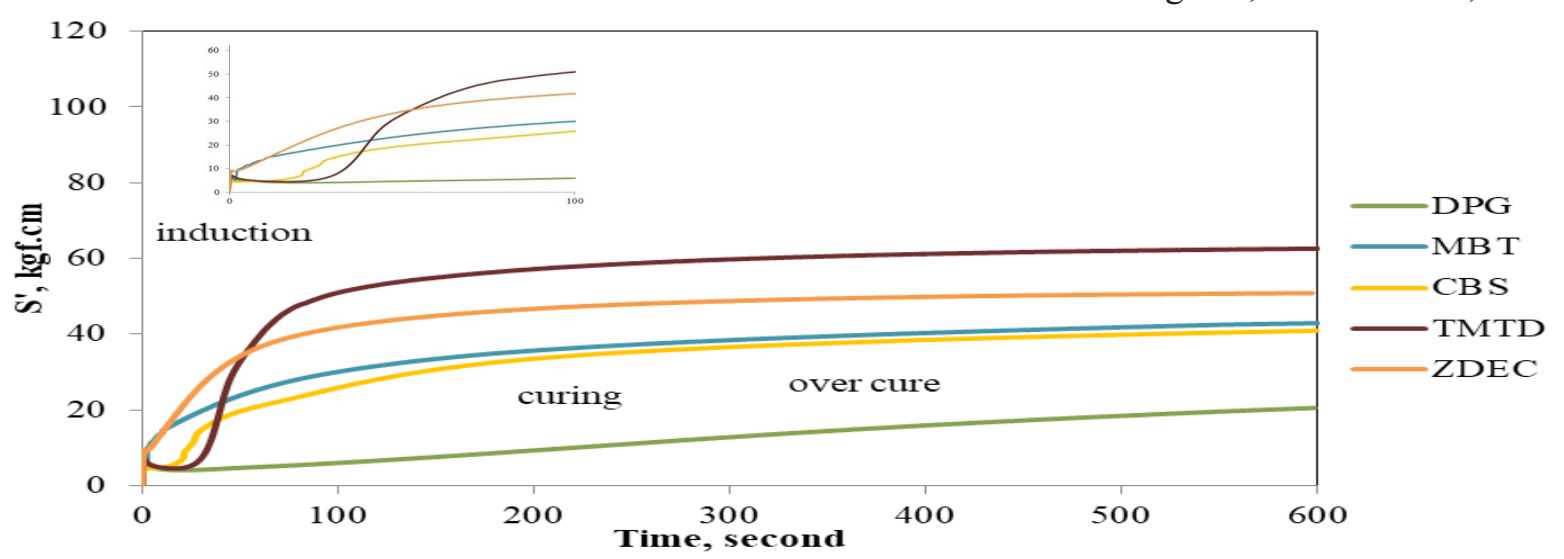

Figure 1. The curing curve of NBR/EPDM compound. 
Table 2. Cure characteristics of NBR/EPDM compound.

\begin{tabular}{ccccccc}
\hline Composite & $\begin{array}{c}\text { S'MH } \\
\text { kgf.cm }\end{array}$ & $\begin{array}{c}\text { S'ML } \\
\text { kgf.cm }\end{array}$ & $\begin{array}{c}\text { S'MH } \\
\text { kgf.cm }\end{array}$ & $\begin{array}{c}\text { S”ML } \\
\text { kgf.cm }\end{array}$ & tan $\delta$ MH & $\begin{array}{c}\text { MH-ML } \\
\text { kgf.cm }\end{array}$ \\
\hline NEM 1 & 20.36 & 4.03 & 6.54 & 3.88 & 0.32 & 16.33 \\
NEM 2 & 43.71 & 5.31 & 11.21 & 5.25 & 0.26 & 38.41 \\
NEM 3 & 40.52 & 4.38 & 11.48 & 4.27 & 0.28 & 36.15 \\
NEM 4 & 62.30 & 4.46 & 14.28 & 4.10 & 0.23 & 57.84 \\
NEM 5 & 50.71 & 9.05 & 12.58 & 6.22 & 0.25 & 41.66 \\
\hline
\end{tabular}

and over cure. It was clearly seen that types of accelerator gave different response. The curing curve of NBR/EPDM was shown in Figure 1.

The different accelerators gave different curing curve of NBR/EPDM as shown in Figure 1. The MBT and CBS was the primary accelerator, while the DPG, TMTD, and ZDEC were the secondary accelerator. The primary accelerator could be used singly or binary. The secondary accelerator usually used to activate the primary accelerator, also can be used singly or binary. Figure 1 shows three stages in curing process, i.e. induction, curing, and over cure. The scorch time $\left(\mathrm{ts}_{2}\right)$, optimum curing time $\left(t_{90}\right)$, and the torque can be obtained from rheometer data. The scorch time and optimum curing time was shown in Figure 2 and the cure characteristics of the NBR/EPDM compound were shown in Table 2.

The first stage, induction stage was the stage before the curing process started. The accelerator took a lot of place in the induction stage. The accelerator could lead the reaction to start sooner. The scorch time showed the safety process time and the end of the induction stage. Figure 2 showed that the DPG has the longest scorch time and optimum curing time, then CBS, TMTD, MBT, and ZDEC.

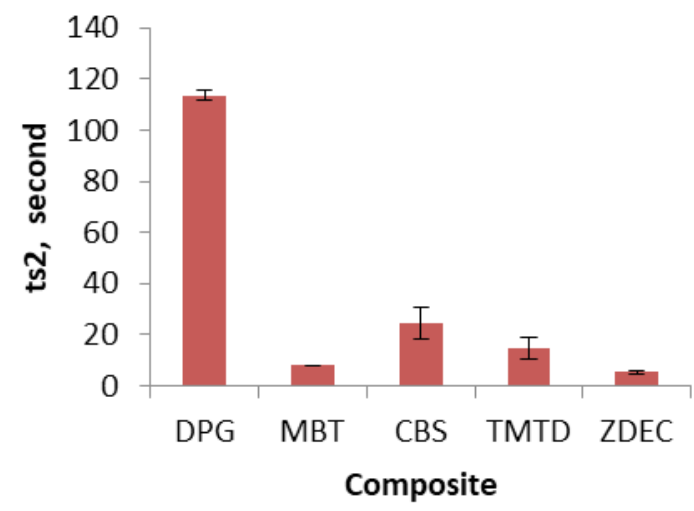

The MBT from thiazole group as the primary accelerator made the shortest scorch time (15 s). MBT has been a primary accelerator with ultrafast processing safety time while the CBS gave the scorch time $24 \mathrm{~s}$. CBS from sulfenamides group had been a primary accelerator but it had fast-delayed action. Hence, the CBS was better than MBT as binary accelerator with secondary accelerator to boost the scorch time.

The ZDEC from dithiocarbamate group and TMTD from thiuram group as secondary accelerator gave medium-fast processing safety time while the DPG as secondary accelerator gave the longest scorch time (516 s). This phenomenon could be happen due to their chemical nature. The DPG from guanidine group was characterized by medium activity. DPG was a slow accelerator and shown in the Figure 1 as a gentle slope. The DPG might be suitable as the binary accelerator combine with the primary accelerator. The other accelerator has two functions i.e. increasing the scorch time because it retards the effect during the curing process and accelerating the crosslink formation because it reacts with zinc oxide (Formela et al., 2015).

The curing stage as second stage was the stage within the formation of crosslinking between

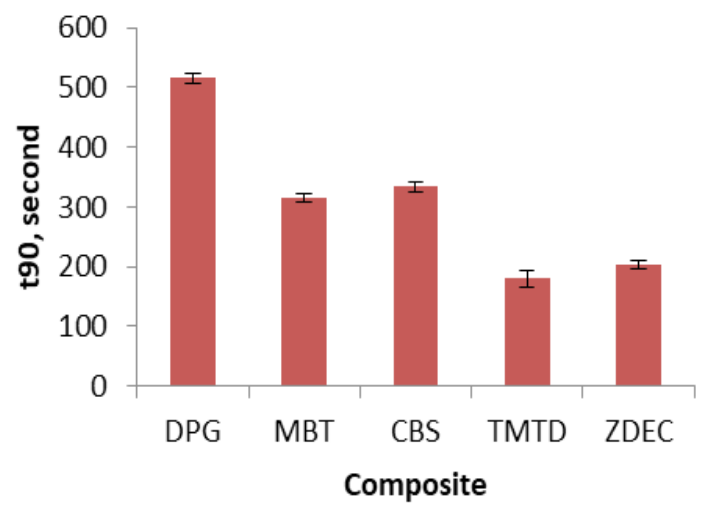

Figure 2. The scorch time (a) and the optimum curing time (b) of NBR/EPDM composite. 
Table 3. The CRI and rate constant $(k)$ of NBR/ EPDM compound.

\begin{tabular}{ccc}
\hline Composite & CRI $\left(\mathrm{min}^{-1}\right)$ & $k\left(\mathrm{~min}^{-1}\right)$ \\
\hline NEM 1 & 0.25 & 0.0033 \\
NEM 2 & 0.33 & 0.0102 \\
NEM 3 & 0.32 & 0.0098 \\
NEM 4 & 0.61 & 0.0389 \\
NEM 5 & 0.50 & 0.0215 \\
\hline
\end{tabular}

rubber-additives. The torque needed and the formation of crosslink for curing stage are shown in Table 2. The torque represents the force needed during the curing process. $M_{H}$ is the maximum torque required within the curing process and $M_{L}$ is the minimum torque required within the curing process. The torque differences could predict the crosslink density of the compounds.

The MBT and CBS, as the primary accelerators, resulted medium crosslink density. The DPG, as secondary accelerator, gave the lowest crosslink density, while TMTD and ZDEC made the high crosslink density. The time needed to cure the rubber was called as optimum curing time. The TMTD and ZDEC, as the secondary accelerators, resulted the fast optimum curing time, while DPG gave the longest optimum curing time. The MBT and CBS, as primary accelerators, yielded the medium curing time. The fastest optimum curing time was suitable for industry because it could reduce the time, electricity, and gave an efficient production.

The maximum torque was related to the increase in stiffness and the shear modulus of the compound (Formela et al., 2015). The accelerator influenced the maximum torque of the compound. Primary accelerators, MBT and CBS, made medium maximum torque. The secondary accelerators, TMTD and ZDEC, gave a high maximum torque, while DPG gave the lowest maximum torque. This result was same with the previous research by Formela et al. (2015). The high maximum torque shows a good synergistic combination between rubber-additives. This maximum torque also relates to crosslink density and optimum curing time. The higher maximum torque tends to the lower curing time.

Cure rate index (CRI) represents the speed of curing process that can be calculated from Equation (1), while the rate constant $(k)$ shows the rate of reaction that can be calculated by plotting Equation (3). The CRI and rate constant are shown in Table 3. The primary accelerators, CBS and MBT, showed medium CRI and rate constant. The secondary accelerator, DPG, made the lowest CRI and also rate constant, while TMTD gave the highest CRI and rate constant. This was related to the optimum curing time where higher CRI and rate constant resulted the fastest optimum curing time. The high CRI and rate constant showed that reaction was fast, thus made the required time for rubber curing was also fast.

The third stage was the over cure stage, it was the stage when the crosslinking almost done. The third stage showed different behavior (equilibrium, reversion, marching) depends on the material characteristics (Fathurrohman et al., 2015). Figure 1 shows that all of the compounds indicated the marching type of curing. The stiffness of the rubber increased along with increasing time. Meanwhile, the reversion showed the decrease in rubber stiffness and the equilibrium showed the same in rubber stiffness.

The damping factor from rheometer data were shown in Table 2. Rubber has viscous and elastic elements, thus it is called viscoelastic material. $S$ ' represents the elastic element of rubber and $\mathrm{S}$ " is the viscous element of rubber. The ratio of viscous and the elastic element were determined as the damping factor $(\tan \delta)$. From Table 2, it was known that the damping factor is related to torque differences (Nabil et al., 2013). The higher $\tan \delta$ $M_{H}$, the lower the crosslinking $\left(M_{H}-M_{L}\right)$. Table 2 shows that the $S^{\prime \prime}$ is lower than $S^{\prime}\left(S^{\prime \prime}<S^{\prime}\right)$. It could be concluded that all compounds have a larger elastic element than viscous element.

\section{Mechanical Properties}

The tensile strength, elongation, and tear strength of the NBR/EPDM composite are shown in Figure 3. The tensile strength describes the load resistance of the composite. The elongation describes the elasticity and flexibility of composite when being stretched. The tear strength describes the resistance of composite against tears. The MBT, as the accelerator, resulted the highest tensile strength, elongation, and tear strength. TMTD gave the lowest tensile strength, elongation, and tear strength. This might be happened because the TMTD composite had high maximum torque, hence led to the decreasing of polymer mobility and the elongation. Meanwhile DPG, CBS, and ZDEC gave the mechanical properties among them. 

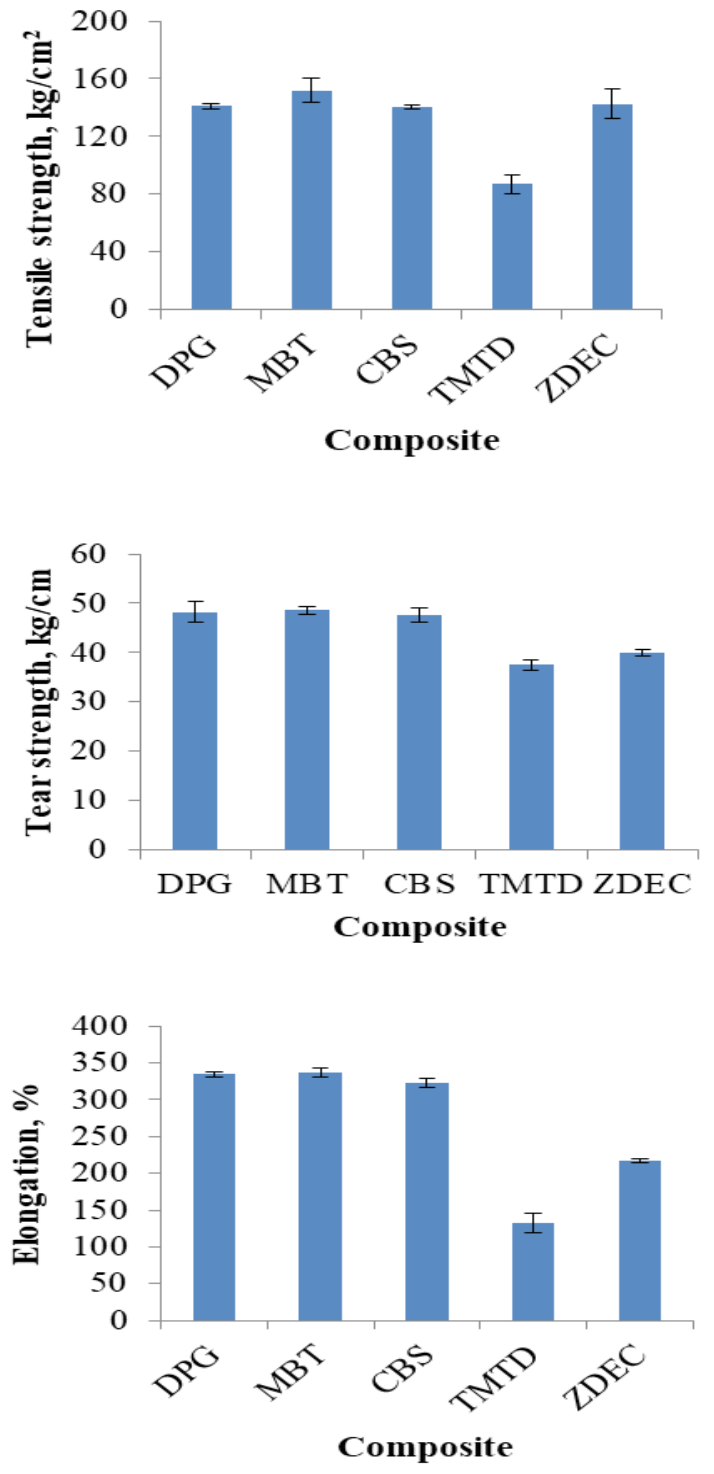

Figure 3. The mechanical properties of NBR/ EPDM composite: tensile strength (a), tear strength (b), elongation (c).

\section{Swelling Property}

The swelling percentage describes the resistance of the composite to the solvent. The poor dispersion of filler in the rubber matrix could led to the void. Hence, there were free volume that can be filled by the solvent (Indrajati et al., 2012). The low solvent resistance could reduce the performance of the composite. The swelling percentage of NBR/EPDM composite with IRM 903 at ambient temperature is presented in Figure 4. All of the blends showed good resistance against solvent (less than $4 \%$ ). The CBS resulted the highest swelling percentage, but not significantly different from other accelerators.

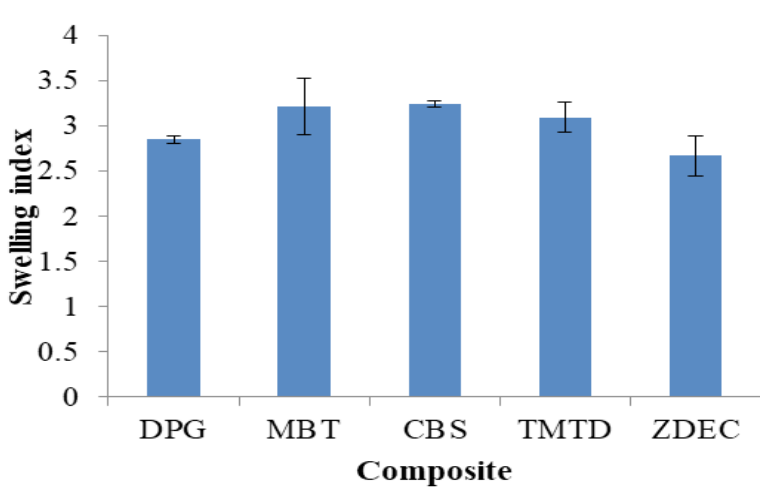

Figure 4. Swelling percentage of NBR/EPDM composite.

\section{CONCLUSIONS}

The NBR/EPDM composite with different accelerator resulted different composite properties. The accelerator made a big role in induction stage and scorch time. The primary accelerator, MBT, gave the fastest scorch time (5 s). The secondary accelerator, DPG, resulted in the longest scorch time (113 s). In the curing stage, the primary accelerators, MBT and CBS, showed the medium optimum curing time, maximum torque, CRI, and rate constant. The secondary accelerators, TMTD and ZDEC, resulted in fast optimum curing time, high maximum torque, CRI, and rate constant. Meanwhile the DPG gave the longest optimum curing time, the lowest maximum torque, CRI, and rate constant. All of the accelerators showed marching type of over cure curing characteristic. The primary accelerator, especially MBT, gave the highest mechanical properties. All of the blends showed good solvent resistance (less than 4\%). From the research, it was known that secondary accelerator, especially TMTD, was a suitable accelerator for improving the curing characteristic properties while the primary accelerator, especially MBT, was a suitable accelerator for improving the mechanical properties of NBR/EPDM composite.

\section{ACKNOWLEDGEMENT}

The authors would like to acknowledge the Centre of Leather, Rubber, and Plastics, Ministry of Industry, Indonesia for financial support.

\section{REFERENCES}

Azar, F. A. N., \& Sen, M. (2017). Effects of accelerator type on stress relaxation behavior and network structure of aged natural rubber/chloroprene rubber vulcanizates. Journal of Elastomers and Plastics, 49(5), 381-396. 
Azizli, M. J., Ziaee, M., Rezaeinia, S., Seyfi, J., Mansourian-Tabaei, M., Hoseinzadeh, M., \& Azizli, M. H. (2018). Studying the roles of nanoclay and blend composition on the improved properties of natural rubber/chloroprene composites. Polymer Composites, 39(5), 15621574. https://doi.org/10.1002/pc. 24100

Bonilla-Cruz, J., Hernández-Mireles, B., MendozaCarrizales, R., Ramírez-Leal,L.A., Torres-Lubián, R., RamosdeValle, L. F., Paul, D. R., \& SaldívarGuerra, E. (2017). Chemical modification of butyl rubber with maleic anhydride via nitroxide chemistry and its application in polymer blends. Polymers, 9(2), 63. https://doi.org/10.3390/ polym9020063

Fathurrohman, M. I., Maspanger, D. R., \& Sutrisno, S. (2015). Vulcanization kinetics and mechanical properties of ethylene propylene diene monomer thermal insulation. Bulletin of Chemical Reaction Engineering \& Catalysis, 10(2), 104-110. https:// doi.org/10.9767/bcrec.10.2.6682.104-110

Formela, K., Wasowicz, D., Formela, M., Hejna, A., \& Haponiuk, J. (2015). Curing characteristics, mechanical and thermal properties of reclaimed ground tire rubber cured with various vulcanizing systems. Iranian Polymer Journal, 24, 289-297. https://doi.org/10.1007/s13726-015-0320-9

Indrajati, I. N., Dewi, I. R., \& Irwanto, D. (2012). Pengaruh variasi rasio HAF/SRF pada vulkanisat NBR. Majalah Kulit, Karet, dan Plastik, 28(2), 59-68. https://doi.org/10.20543/mkkp.v28i2.106

Jovanović, V., Samaržija-Jovanović, S., BudinskiSimendić, J., Marković, G., \& MarinovićCincović, M. (2013). Composites based on carbon black reinforced NBR/EPDM rubber blends. Composites Part B: Engineering, 45(1), 333-340. $\quad$ https://doi.org/10.1016/j. compositesb.2012.05.020

Kapgate, B. P., \& Das, C. (2014). Reinforcing efficiency and compatibilizing effect of sol-gel derived in situ silica for natural rubber/chloroprene rubber blends. RSC Advances, 4(102), 58816-58825. https://doi.org/10.1039/c4ra09415h

Mayasari, H. E., \& Setyadewi, N. M. (2018). Thermogravimetry and swelling characteristics of NBR/EPDM blends with some compatibilizers. AIP Conference Proceedings, 2049, 020042. https://doi.org/10.1063/1.5082447

Mayasari, H. E., Setyorini, I., \& Yuniari, A. (2019). The blending of EPDM/NR with maleic anhydride as compatibilizer: Comparing the effect of accelerators on cure characteristic and mechanical properties. Indonesian Journal of Chemistry, 19(1), 106-114. $\quad$ https://doi.org/10.22146/ ijc. 27730

Movahed, S. O., Ansarifar, A., \& Mirzaie, F. (2015). Effect of various efficient vulcanization cure systems on the compression set of a nitrile rubber filled with different fillers. Journal of Applied Polymer Science, 132(8), 1-10. https://doi. org/10.1002/app.41512

Muniyadi, M., \& Ismail, H. (2013). Curing, tensile and morphological properties of treated bentonite filled EPDM composite. Advanced Materials Research, 844, 285-288. https://doi.org/10.4028/ www.scientific.net/AMR.844.285

Nabil, H., Ismail, H., \& Azura, A. R. (2013). Compounding, mechanical and morphological properties of carbon-black-filled natural rubber/ recycled ethylene-propylene-diene-monomer (NR/R-EPDM) blends. Polymer Testing, 32(2), 385-393. $\quad$ https://doi.org/10.1016/j. polymertesting.2012.11.003

Nabil, H., Ismail, H., \& Azura, A. R. (2014). Optimisation of accelerators and vulcanising systems on thermal stability of natural rubber/ recycled ethylene-propylene-diene-monomer blends. Materials \& Design, 53, 651-661. https:// doi.org/10.1016/j.matdes.2013.06.078

Nampitch, T., \& Buakaew, P. (2006). The effect of curing parameters on the mechanical properties of styrene-NR elastomers containing natural rubber-graft-polystyrene. Kasetsart Journal: Natural Science, 40(6), 7-16.

Nik Ismail, N. I., Ansarifar, M. A., \& Song, M. (2014). Improving heat ageing and thermal properties of silicone rubber using montmorillonite clay. Journal of Applied Polymer Science, 131, 41061. https://doi.org/10.1002/app.41061

Sun, C. H., Gu, Z., Li, P. Y., Song, G. J., \& Shan, C. P. (2013). The preparation and properties of chloroprene rubber (CR)/organic montmorillonite (OMMT) nanocomposites. Advanced Materials Research, 742, 147-151. https://doi.org/10.4028/ www.scientific.net/AMR.742.147

Yi, Z., Jincheng, W., Youcheng, D., Chen, P., \& Wen, Z. (2014). Enhanced application properties of EPDM/montmorillonite composites. Polymers and Polymer Composites, 22(9), 799-808. 
